\title{
Laurent Bourdeau, Maria Gravari-Barbas et Mike Robinson (dir.), Tourisme et patrimoine mondial
}

Coll. "Patrimoine en mouvement", Presses universitaires de Laval, mars 2012, 320 pages

\section{Philippe Violier}

\section{OpenEdition Journals}

Édition électronique

URL : http://journals.openedition.org/tourisme/101

DOI : 10.4000/tourisme.101

ISSN : 2492-7503

\section{Éditeur}

Éditions touristiques européennes

\section{Édition imprimée}

Date de publication : 1 décembre 2013

Pagination : 80-82

ISSN : 2109-5671

\section{Référence électronique}

Philippe Violier, « Laurent Bourdeau, Maria Gravari-Barbas et Mike Robinson (dir.), Tourisme et patrimoine mondial », Mondes du Tourisme [En ligne], 8| 2013, mis en ligne le 01 juin 2015, consulté le 22 septembre 2020. URL : http://journals.openedition.org/tourisme/101 ; DOI : https://doi.org/ 10.4000/tourisme.101

Ce document a été généré automatiquement le 22 septembre 2020

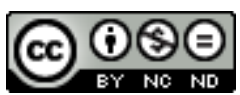

Mondes du tourisme est mis à disposition selon les termes de la licence Creative Commons Attribution - Pas d'Utilisation Commerciale - Pas de Modification 4.0 International. 


\section{Laurent Bourdeau, Maria Gravari- Barbas et Mike Robinson (dir.), Tourisme et patrimoine mondial}

Coll. "Patrimoine en mouvement", Presses universitaires de Laval, mars 2012, 320 pages

\section{Philippe Violier}

\section{RÉFÉRENCE}

Laurent Bourdeau, Maria Gravari-Barbas et Mike Robinson (dir.), Tourisme et patrimoine mondial, coll. "Patrimoine en mouvement", Presses universitaires de Laval, 2012.

1 Cet ouvrage rassemble dix-sept textes sur le thème des relations entre le tourisme et le patrimoine. Il est structuré en trois parties. La première traite du sens et des valeurs accordées au patrimoine mondial à travers les expériences et le vécu de différentes populations. La seconde aborde le rôle des acteurs. La troisième analyse l'utilisation du label pour le développement économique et touristique.

2 Une solide introduction, due aux directeurs, propose un changement de paradigme dans l'analyse des rapports entre tourisme et patrimoine mondial, à savoir appréhender le tourisme comme un phénomène endogène consubstantiel de la nature même de cet objet. Il nous semble que cette proposition est déjà exprimée par Olivier Lazzarotti, ouvrage d'ailleurs cité. Le nouvel angle serait alors de circonscrire l'analyse au patrimoine mondial, distinction qui relève d'experts et dont l'universalité est une construction en marche. Les auteurs abordent le tourisme avec subtilité et s'inscrivent en faux contre les trop fréquentes condamnations auxquelles le tourisme est soumis dès qu'il s'agit de patrimoine. Ils relèvent notamment que, dans la liste du Patrimoine mondial en péril, le tourisme n'est qu'une cause marginale. Cependant nous ne pouvons que déplorer la superficialité de l'approche du tourisme. Nous relevons, par exemple, 
que le concept est abordé comme un système, certes, mais qu'il manque l'essentiel, à savoir la finalité, la "recréation", qui n'est pas précisée. Par ailleurs n'y a-t-il pas une contradiction dans l'affirmation de la consubstantialité et le constat de l'inégale fréquentation des sites? De fait, l'inscription est un jeu entre experts et acteurs : ils visent la promotion de certains lieux, sans réellement prendre en compte les aspirations de touristes - des touristes dont les intérêts sont d'autant plus variés que, désormais, les différentes sociétés du monde accèdent à cette pratique. Du coup, parfois ça marche, parfois non. Enfin, plusieurs auteurs abordent la question des habitants, de leurs représentations du patrimoine et de leurs relations avec les touristes, mais cette question n'apparaît ni dans l'introduction, ni dans le titre de l'ouvrage.

Dans la première partie, le texte de Mathieu Dormaels souffre également d'une approche un peu rapide de la littérature dans le champ du tourisme. L'auteur pointe notamment que dans la définition du tourisme manque la notion de "présence physique dans les lieux" (p.25), pourtant contenue dans le déplacement, et reprend le mot récréation, bien pauvre, au lieu de celui de "recréation", porteur de sens. Dans leur article, "La perception d'authenticité d'un site touristique classé au patrimoine mondial de l'Unesco", Sandra Camus et Jean-Michel Sahut proposent d'identifier ce qu'est l'authenticité pour les touristes. Ils procèdent par entretiens pour recueillir l'expérience vécue par les touristes dans douze sites. Malgré l'absence d'explicitation de cette sélection et, au-delà de l'intérêt d'interroger des personnes qui n'ont pas visité tous les sites - grâce à la technique dite "projective du rêve éveillé" (p. 41), laquelle tente de pallier la grande difficulté de constituer un panel d'individus ayant visité le même échantillon de lieux-, l'analyse conclut sur la capacité du touriste à passer des compromis pour passer outre les trois paradoxes identifiés par les auteurs, à savoir l'originalité, la non-dénaturation et la non-marchandisation. Se dégagent alors des principes d'action: transformer de manière modérée; favoriser la qualité de l'expérience vécue ; activer le sentiment de nostalgie ; distinguer clairement entre ce qui relève du patrimoine et ce qui dépend de la valorisation. Le texte de Wided Majdoub est décevant par la place occupée par la revue de littérature sur l'expérience, au détriment de l'étude de cas de la médina de Sousse, peu visitée selon l'auteur. Les conclusions intéressantes que l'auteur retire de vingt entretiens auraient mérité d'être davantage discutées, notamment en relation avec le capital social des individus interrogés. Maria Gravari-Barbas et Cécile Renard se proposent d'étudier la contribution du classement à la patrimonialisation de l'architecture moderne, à partir du cas de la ville du Havre caractérisé par une mobilisation très active des acteurs légitimes afin de changer l'image de leur ville. L'approche est intéressante mais la conclusion, qui repose sur l'analyse d'un seul guide, semble hâtive. Les autres éléments avancés (appartement témoin, centre d'interprétation, signalétique) témoignent bien de la volonté politique de mise en tourisme, mais pas d'un changement du regard des populations non résidentes sur la ville. Ensuite, Florence Revelin et Marie Roué proposent une analyse très fouillée du site Laponia, au nord de la Suède. À partir d'entretiens, elles montrent que la volonté de classement relevait de stratégies différenciées entre les Suédois, valorisant le paysage naturel, et les Samis, cherchant au contraire à promouvoir leur identité culturelle. De leur côté, les touristes sont à la recherche d'une nature sauvage mais sont aussi partagés : ceux originaires de la région sont les seuls à savoir que le site est classé, au contraire des touristes suédois ou internationaux. 
4 La seconde partie met l'accent sur les acteurs. Le premier texte, signé par Sarah Russeil, pose la question de "la place et [du] rôle de l'organisation des villes du patrimoine mondial dans la gestion des biens inscrits". Pour traiter cette question, l'auteur analyse les discours écrits sur l'histoire de cette organisation et réalise deux études de cas, Québec et Lyon, traitées par des entretiens. Elle conclut que la place des villes s'est accrue et que cette promotion est en lien avec le développement de la valorisation touristique du classement. Sandra Guinand montre comment le classement sur la liste du patrimoine mondial du quartier de Ribeira, à Porto (Portugal), est venu légitimer une reconnaissance acquise nationalement. La labellisation a induit un développement touristique, alors que la réhabilitation antérieure visait l'amélioration du logement des habitants. Du fait de ces objectifs différents, l'auteur pose la question de la cohabitation entre touristes, qui recherchent l'“authenticité", et habitants, qui aspirent à une certaine modernité. Pierre Sintès montre comment la patrimonialisation réveille les tensions anciennes entre les différentes populations qui ont habité l'île de Rhodes.

5 La troisième section aborde donc l'utilisation du label dans la valorisation touristique. Elle débute par une analyse réalisée par Géraldine Djament-Tran, Édith Fagnoni et Sébastien Jacquot. Les auteurs ont confronté les arguments élaborés par le niveau local et les reformulations élaborées par les experts lors des procédures de classement des sites, d'une part, aux modalités de mise en tourisme, d'autre part. Pour cela, ils croisent une analyse quantitative et une étude qualitative à partir de trois cas sélectionnés, sans que ces choix ne soient justifiés. La finalité de l'exercice n'apparaît pas bien, sauf que les modalités du tourisme et les raisons des classements ne sont pas connectées. Terence Keller Andrade abonde dans le même sens en montrant que la labellisation du parc national de Jaú, en haute Amazonie brésilienne, n'a pas induit de flux touristiques significatifs et que le label n'est pas utilisé. De leur côté, Pascal Marcotte et Laurent Bourdeau montrent, par une analyse systématique menée à partir des sites internet des institutions touristiques, que le label du patrimoine mondial est d'autant plus utilisé que le classement est récent, soit entre deux et dix ans. Nadia Belaidi, quant à elle, souligne que le classement de la montagne de la Table, au Cap (Afrique du Sud), est surtout utilisé pour construire la démocratie après l'apartheid.

Cet ouvrage riche et dense ouvre de nombreuses réflexions. Nous soupçonnons qu'il s'agisse d'actes d'un colloque ou d'un séminaire; ce n'est pas explicité. Pour ce type d'ouvrage, toujours très difficile à mettre en cohérence et à construire, c'est plutôt une réussite.

\section{AUTEURS}

\section{PHILIPPE VIOLIER}

Université d'Angers 